
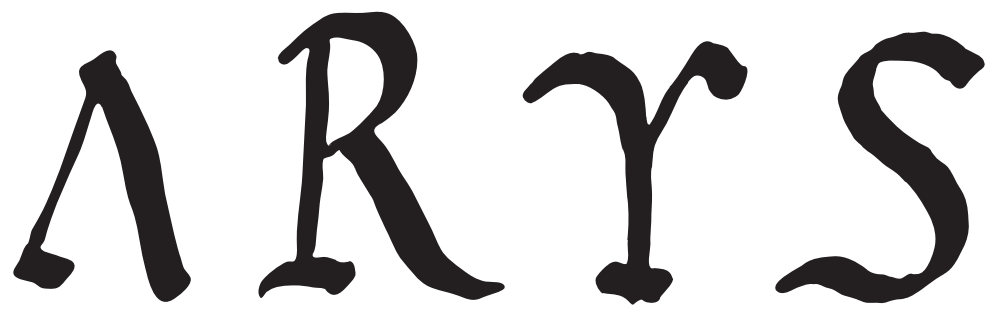

Antigüedad, Religiones y Sociedades

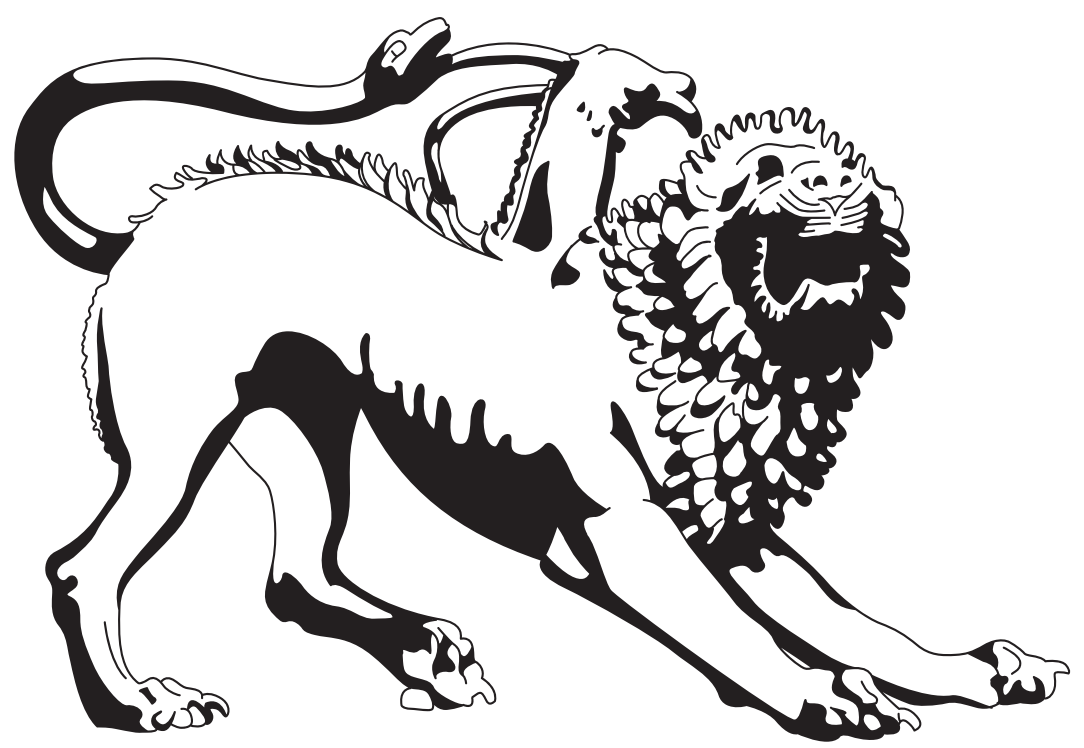

VERENDA NUMINA: TEMOR Y EXPERIENCIA RELIGIOSA EN EL MUNDO ANTIGUO 
DIRECTOR

Jaime Alvar EzQuerra

(Universidad Carlos III de Madrid)

\section{COMITÉ CIENTÍFICO \\ Radu Ardevan \\ (Universidad Babeş-Bolyai, Cluj-Napoca) JUDY BARRINGER \\ (University of Edimburg) Nicole Belayche \\ (École Pratique des hautes études à Paris) CORInne Bonnet \\ (Université Toulouse II) \\ Antonio GonZales \\ (Univ. Franche Comte) \\ María José Hidalgo de la Vega \\ (Universidad de Salamanca) \\ Rita LizzI \\ (Universita degli Studi di Peruggia) \\ Francisco Marco Simón \\ (Universidad de Zaragoza) JoHN NORTH \\ (University College London) \\ Domingo Plácido SuÁrez \\ (Universidad Complutense de Madrid) \\ Mario Torelli \\ (Università della Calabria, Cosenza; \\ Accademia Nazionale dei Lincei) \\ Henk S. Versnel \\ (University of Leiden)}

DISEÑO Y MAQUETACIÓN

Syntagmas (www.syntagmas.com)

\section{EDITA}

Dykinson S.L. (www.dykinson.com) Instituto de Historiografía Julio Caro Baroja

Universidad Carlos III de Madrid Asociación ARYS

\section{SUBSCRIPCIONES}

El precio anual de la subscripción es de $18 €$ (individual) y $30 €$ (instituciones). Para subscripciones fuera de España el precio es de $30 \$$ (individual) y $50 \$$ (instituciones).

Toda la correspondencia para subscripción, permisos de publicación, cambios de dirección $\mathrm{y}$ cualquier otro asunto debe dirigirse a:
SECRETARIO

JuAn RAMÓN CARbó García

(Universidad Católica San Antonio de Murcia)

CONSEJO DE REDACCIÓN

María del Mar Marcos Sánchez

(Universidad de Cantabria)

José Ignacio SAn Vicente GonZÁlez

De Aspuru

(Universidad de Oviedo)

Adolfo Domínguez Monedero

(Universidad Autónoma de Madrid)

ANA IrIARTE

(Universidad del País Vasco)

Rebeca Rubio Rivera

(Universidad de Castilla La Mancha)

José LuIs López CAstro

(Universidad de Almería)

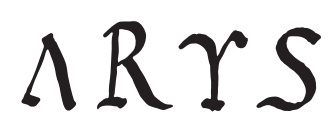

Volumen 14- 2016 - ISSN: 1575-166X

Depósito Legal M-32333-2014

ARYS: Antigüedad, Religiones y Sociedades figura indizada en CIRC, Dialnet, DICE, ERIH PLUS, Interclassica, Latindex, MIAR, RESH. Sobre la política de la revista e instrucciones para los autores, ver últimas páginas del volumen.

Reservados todos los derechos.

No se pueden hacer copias por ningún procedimiento electrónico o mecánico,

incluyendo fotocopias, o grabación magnética o cualquier almacenamiento de información y sistemas de recuperación sin permiso escrito de los escritores.

Revista ARYs

Biblioteca de la facultad de Humanidades

Universidad Carlos III de Madrid

C/ Madrid, 135

28903 Getafe (Madrid) ESPAÑA

E-Mail: imuro@db.uc3m.es

Tlfno: 916249207 


\section{$\Lambda R \Upsilon S$}

NúMERO $14-2016$

\section{VERENDA NUMINA: TEMOR Y EXPERIENCIA}

RELIGIOSA EN EL MUNDO ANTIGUO

\section{PRÓLOGO}

7 TEMOR Y EXPERIENCIA RELIGIOSA EN EL MUNDO ANTIGUO:

A MODO DE PRÓLOGO

Silvia Alfayé Villa (Universidad de Zaragoza)

\section{MONOGRÁFICO}

23 EL MIEdo Y LA RELIGIÓN: ALGUNAS REFLEXIONES GENERALES

Francisco Diez de Velasco (Universidad de La Laguna)

43 CULTO Y RITO EN CUEVAS: MODELOS TERRITORIALES DE VIVENCIA Y EXPERIMENTACIÓN DE LO SAGRADO, MÁS ALLÁ DE LA MATERIALIDAD (SS. V-II A.N.E.)

Carmen Rueda Galán y Juan Pedro Bellón Ruiz (Universidad de Jaén) 
81 CAPUT IN IECORE NON FUIT. LA 'CABEZA' DE LOS CÓNSUles POR LA SALVACIÓN DE LA REPÚBLICA

José A. Delgado Delgado (Universidad de La Laguna)

109 LOS TEMORES DEL MAGO: MIEDOS EN TORNO A LA ACCiÓN MÁGICA EN LA ANTIGUA Roma

Silvia Alfayé Villa (Universidad de Zaragoza)

153 FEARSCAPES CRISTIANOS EN EL EgIPTO TARDOANTIGUO

Clelia Martínez Maza (Universidad de Málaga)

171 Xibalba, el Lugar del Miedo: las cuevas y el inframundo de los ANTIGUOS MAYAS

Holley Moyes (University of California, Merced))

\section{VARIA}

193 UNA REFLEXIÓN SOBRE EL ORIGEN DE LA IMPORTANCIA DE LA MÚSICA EN la antigua Mesopotamia a partir de los Cilindros de Gudea A y B Daniel Sánchez Muñoz (Universidad de Granada)

\section{RECENSIONES}

223 McIntyre, Gwynaeth: A Family of Gods: The Worship of the Imperial Family in the Latin West. University of Michigan Press, Ann Arbor, 2016. Carmen Alarcón Hernández (Universidad de Sevilla) 


\title{
TEMOR Y EXPERIENCIA RELIGIOSA EN EL MUNDO ANTIGUO: A MODO DE PRÓLOGO ${ }^{1}$
}

FEAR AND RELIGIOUS EXPERIENCE IN THE ANCIENT WORLD: A PROLOGUE

\author{
Silvia Alfayé Villa \\ Universidad de Zaragoza \\ alfaye@unizar.es
}

HaCe mÁs de un Siglo, S. Freud escribió: "los dioses cumplen una triple función: exorcizan los terrores naturales, nos reconcilian con la crueldad del destino, especialmente cuando se manifiesta en la muerte, y nos resarcen de los sufrimientos y las privaciones que impone al hombre la vida civilizada en común"2. Las contribuciones de este número monográfico dedicado a "Verenda Numina: temor y experiencia religiosa en el mundo antiguo", ofrecen diversas aproximaciones a las complejas, dinámicas y polifacéticas relaciones entre los miedos individuales y colectivos, las expresiones religiosas, las emociones y las prácticas rituales en varias sociedades del mundo anti-

1. Este trabajo se ha realizado dentro del proyecto de investigación "Verenda Numina: temor y experiencia religiosa en el mundo antiguo” (JIUZ-2014-HUM-01), financiado por la Universidad de Zaragoza y dirigido por la autora.

2. Cit. en MONGARDINI, C.: Miedo y sociedad, Madrid, 2007, 40. 
guo $^{3}$. El análisis de la interrelación y retroalimentación entre el temor y la experiencia religiosa en la Antigüedad es una línea de investigación reciente que se integra dentro de los estudios sobre el miedo en la Historia o Fobología, pero también dentro de la Emocionología, dedicada a la comprensión de la vida emocional de las sociedades del pasado ${ }^{5}$, y de la denominada Arqueología de los Sentidos ${ }^{6}$. Sin embargo, debido al carácter novedoso de la Fobología antigua como tendencia historiográfica, aún se están perfilando las metodologías de análisis adecuadas para aprehender los diversos rostros del espanto en la Antigüedad y, salvo recientes excepciones, son relativamente escasos los trabajos específicos que exploran las relaciones entre las experiencias religiosas y los temores de las culturas antiguas ${ }^{7}$.

3. Sobre la relación entre temor y religión, cf., entre otros, DÍEZ DE VELASCO, F.: "El miedo y la religión: reflexiones teóricas y metodológicas", DÍEZ DE VELASCO, F. (ed.): Miedo y religión, Madrid, 2002, 367-380; DÍEZ DE VELASCO, F. (ed.): Miedo y religión, Madrid, 2002; LINCOLN, B.: Holy Terrors: Thinking about Religion after September 11, Chicago, 2003; NUSSBAUM, M.C.: La nueva intolerancia religiosa. Cómo superar la politica del miedo en una época de inseguridad, Barcelona, 2013.

4. Así, por ejemplo, NEWMAN, P.: A history of terror. Fear \& Dread through the ages, Stroud, 2000; BAUMANN, Z.: Miedo líquido. La sociedad contemporánea y sus temores, Barcelona, 2006; BOURKE, J: Fear. A cultural history, London, 2006; MONGARDINI: Miedo...; LINKE, U., y SMITH, D.T. (EDS.): Cultures of Fear. A critical reader, New York, 2009; KORSTANJE, M.E.: "La fobología, ¿ciencia o forma de entretenimiento?", Nómadas. Revista Crítica de Ciencias Sociales y Jurídicas, 31, 3, 2011, 175-189; DELUMEAU, J.: El miedo en Occidente (siglos XIV-XVIII). Una ciudad sitiada, Madrid, 2012; LAFFLAN, M., y WEISS, M. (EDS.): Facing Fear. The History of an Emotion in Global Perspective, Princeton-Oxford, 2012; LEYS, R.: "How Did Fear Become a Scientific Object and What Kind of Object Is It?", PAMPLER, J., y LAZIER, B. (eds.): Fear across the disciplines, Pittsburgh, 2012, 51-77; PAMPLER, J., y LAZIER, B. (EDS.): Fear across the disciplines, Pittsburgh, 2012; BORRERO, M. ET AL.: El miedo en la Historia, Valladolid, 2013; BOSCOBOINIK, A., y HORÁKOVA, H. (EDS.): The Anthropology of Fear. Cultures beyond Emotions, Münster, 2014; PERNAU, M.: “What Object is Fear?”, History \& Theory, 54, 1, 2015, 86-95.

5. Vid., por ejemplo, CHANIOTIS, A. (ED.): Unveiling Emotions. Sources and Methods for the Study of Emotions in the Greek World, Stuttgart, 2012; MICHAELS, A., y WULF, C. (EDS.): Emotions in rituals and performances, London-New York, 2012; CHANIOTIS, A., y DUCREY, P. (EDS.): Unveiling Emotions II. Emotions in Greece and Rome: texts, images, material culture, Stuttgart, 2013.

6. CHANIOTIS, A., ET AL. (EDS.): Body, Performance, Agency, and Experience. Ritual Dynamics and the Science of Ritual. II, Wiesbaden, 2010; HAMILAKIS, Y.: "Archaeology of the senses", INSOLL, T. (ed.), The Oxford Handbook of the Archaeology of Ritual and Religion, Oxford, 2011, 208-225; ID., Arqueología de los sentidos. Experiencia, memoria y afecto, Madrid, 2015.

7. DODDS, E.R.: Los griegos y lo irracional, Madrid, 1997; RUBEL, A.: Fear and Loathing in Ancient Athens: Religion and Politics during the Peloponnesian War, London-New York, 2014; COIN-LONGERAY, S., y VALLERAT, D. (EDS.): Peurs Antiques, Saint-Étienne, 2015; PATERA, M.: Figures grecques de lépouvante de l'antiquité au présent. Peurs enfantines et adultes, Leiden-Boston, 2015; DUMITRU, M.L., HALICHIAS, A.C., y POPA, N.A. (EDS.): Expressions of Fear from Antiquity to the Contemporary World, Newcastle-upon-Tyne, 2016; ALFAYÉ, S.: Homo Timens: miedo, magia y ritual en la antigua Roma, e.p. 
Sobre estas bases, los artículos que componen este número monográfico de Arys ofrecen diferentes miradas y perspectivas sobre los miedos, las experiencias religiosas y las prácticas rituales en ámbitos culturales, espaciales y cronológicos tan dispares como el mundo ibérico de los siglos V-II a.C., el Egipto tardoantiguo, la cultura maya del período clásico, la antigua Roma de las épocas republicana e imperial, e incluso nuestro presente post-moderno. Para explorar el poliédrico binomio “miedo y religión" en esos horizontes culturales, los autores han recurrido al uso interdisciplinar de fuentes y metodologías diversas, desarrollando en sus trabajos historias parciales sobre aspectos concretos del miedo asociados a sistemas religiosos del mundo antiguo. Es por ello que este volumen monográfico no constituye una síntesis ni una "historia" narrativa de la relación entre la experiencia religiosa y el temor en la Antigüedad -hasta la fecha inexistente-, sino que muestra, a través de diversos estudios de caso, las posibilidades de una novedosa y prometedora línea de investigación, y plantea nuevos caminos por explorar para seguir profundizando en el conocimiento de los miedos y las religiones del pasado, un tema infravalorado por la historiografía pese a que, ya en el siglo I d.C., Estacio, Tebaida, III, 661, señalara la importancia del miedo como hacedor de dioses (y religiones): primas in orbe deos fecit timor.

Las contribuciones a "Verenda Numina" comienzan con la personal aproximación al tema que plantea F. Díez de Velasco, un investigador pionero en el estudio de la relación entre temor y experiencia religiosa dado que hace más de una década, en el año 2002, fue el editor de la obra "Miedo y religión”, hoy convertida en una referencia obligada. En su artículo sobre "El miedo y la religión: algunas reflexiones generales”, explora las diversas combinaciones de esos elementos en el devenir humano, y revisa críticamente las tendencias historiográficas que se han ocupado de su estudio desde la perspectiva de la Historia de las Religiones. Además, reflexiona sobre la intolerancia religiosa y el recelo que suscita el fenómeno religioso en la Postmodernidad, y sobre las supuestas "religiones del miedo" y su uso como componente ideológico de las actuales "políticas del miedo" y "geopolíticas de la emoción"8, entre otras interesantes cuestiones, como la del debate historiográfico irresuelto sobre el origen de la religión y su relación con el temor a la muerte y/o la visión aterradora de lo sagrado 9 . Así, un sector académico considera que el origen de los dioses y de la religión sería precisamente el temor reverencial suscitado por la contemplación y el contacto con lo sagrado, por la experiencia de lo numinoso, en forma de sobrecogedoras apariciones sobrenaturales y fenómenos naturales aterradores, como expresa Lucrecio, De rerum natura V, 1217-1220: “¿A quién el ánimo no se le encoge de temor a los dioses, a quién

8. MOISI, D.: The Geopolitics of Emotion. How cultures of fear, humiliation and hope are reshaping the world, New York, 2010.

9. Vid. DENNETT, D.C.: Romper el hechizo. La religión como fenómeno natural, Buenos Aires, 2007. 
no se le desmayan de miedo las carnes cuando la tierra chamuscada por el espantoso rayo retiembla y los truenos recorren el cielo?"10. Significativamente, en la antigua Grecia el término phriké, "escalofrío, estremecimiento", designaría tanto una reacción fisiológica instintiva del miedo, como una metonimia para la emoción como respuesta primaria a la contemplación visual de una manifestación sagrada ${ }^{11}$. De acuerdo con esa teoría, sería en ese contexto emocional de pánico ante las verenda numina cuando surgirían la religión como narrativa de la racionalidad humana que daría sentido a esa experiencia trascendente, y el ritual como estrategia tecnológica que permitía aplacar y controlar a las poderosas y peligrosas potencias sobrenaturales. Y es que, como señala R. Pannikar, "la religión con sus cultos y creencias proporciona al homo sapiens una solución o por lo menos una coraza para defenderse y en cierta manera racionalizar la angustia de su existencia precaria. Frente al misterio delante de lo desconocido, la religión ofrece una cierta seguridad y un conjunto de explicaciones más o menos convincentes" ${ }^{\prime 2}$.

Sin embargo, como analiza F. Díez de Velasco en su contribución, otra tendencia historiográfica identifica el temor a la muerte (terror mortis) como el miedo primordial del hombre, y como el origen emocional y el elemento fundamental del sentimiento religioso ${ }^{13}$. De acuerdo con la "hipótesis del tanatismo", somos la única especie animal consciente de su propia mortalidad, y esa certeza nos resulta tan insoportable, tan aterradora y angustiosa, que necesitamos dar sentido a la frágil y corta existencia humana, encontrar un horizonte metafísico que trascienda lo inevitable y nos permita vivir con la ficción de la inmortalidad, entendida como no-finitud. Desde los presupuestos de la "teoría de gestión del terror" ${ }^{14}$, que sitúa al terror mortis en el centro de una teoría general del comportamiento humano, uno de los mecanismos para controlar ese miedo sería el desarrollo de una cosmovisión cultural con un discurso religioso que explique el aquende y ofrezca una tranquilizadora posibilidad de allende, disminuyendo así la angustia vital del homo timens ante el abismo de la

10. Trad. de Socas 2003. Sobre la relación entre miedo y religión en Lucrecio, cf., VON ALBRETCH, M.: “Terror et Pavor: politica e religione in Lucrezio", URSO, G. (ed.), Terror et pavor. Violenzia, intimidazione, clandestinità nel mondo antico, Pisa, 2006, 231-245.

11. CAIRNS, D.: "A short story of shudders", CHANIOTIS, A., y DUCREY, P. (EDS.): Unveiling Emotions II. Emotions in Greece and Rome: texts, images, material culture, Stuttgart, 2013, 85-108 (93-105).

12. PANNIKAR, R.: "Presentación: miedo, religión y milenio", DÍEZ DE VELASCO (ED.): Miedo y religión, Madrid, 2002, 9-13 (9).

13. Cf., entre otros, DÍEZ DE VELASCO (ED.): Miedo y religión...; EDWARDS, C.: Death in Ancient Rome, New Heaven-London, 2007, 78-112; ESLER, P.F.: "La muerte de Jesús y el Siervo Sufriente de Isaías: aproximación psico-sociológica a una cuestión histórica y teológica”, BERNABÉ, C., y GIL, C. (eds.): Reimaginando los orígenes del cristianismo, Estella, 2008, 171-191; ARIES, A.: El hombre ante la muerte, Madrid, 2011; DELUMEAU: El miedo en Occidente.

14. ESLER: “La muerte de Jesús...", 174-175. 
no-existencia, como describieran Lucrecio, De rerum natura, III, 37-93, y Tertuliano, De anima, IV, 9-10.

Pero sean cuales sean los elementos ansiógenos que dieron origen a las religiones primigenias, es indudable que los miedos no son solo reacciones fisiológicas y objetivas a una amenaza, sino construcciones culturales y situacionales, respuestas subjetivas y sociales insertas en una temporalidad, una materialidad, una espacialidad, y un contexto histórico y biográfico determinado. Es decir, cada individuo y cada cultura posee -y padece-sus propios temas amenazantes y sus figuras fantasmáticas, que son resultado de su imaginación sociológica y de la forma-de-estar-en-el-mundo de esa persona y de ese colectivo ${ }^{15}$. Y conforme a ellas, y sobre la base de la creencia en la existencia de dioses y espíritus que pueden intervenir en los asuntos humanos, los agentes desarrollarán y seleccionarán estrategias específicas para gestionar sus temores e incertidumbres, entre las que se incluyen el pensamiento mágico y la solicitud de la intervención sobrenatural a través del ritual; es decir, la religión y la magia ${ }^{16}$.

Ambas son formas culturales de racionalidad humana y tecnologías de supervivencia desarrolladas por nuestra especie para afrontar las dificultades de la vida y transformar la realidad a conveniencia, que se rigen por su propia lógica interna, y que se basan sobre el presupuesto de que a través de la ejecución correcta de los rituales mágicos y/o religiosos es posible obtener ayuda sobrenatural y manipular la realidad en nuestro beneficio, en lo que B. Malinowski denominó "una ritualización del optimismo humano"17. De esta forma, la práctica del rito provoca en el actor la neutralización de su angustia y su impotencia en tanto que le permite expresar un deseo -y, como señalara L. Wittgenstein, "la representación de un deseo es, eo ipso, la representación de su satisfacción"18 -, devolviendo así al ser humano la capacidad agéntica sobre su propia realidad, restaurando la sensación de control y la ilusión de predictibilidad sobre un entorno incierto, reforzando su seguridad ontológica, y ofreciéndole protección y consuelo frente al infortunio, entre otros beneficios ${ }^{19}$. Como

15. ALFAYÉ: Homo timens..., e.p.

16. No es este el contexto para profundizar en el debate académico irresuelto sobre religión y magia en la Antigüedad; cf., por ejemplo, OTTO, B.C., y STAUSBERG, M. (EDS.): Defining Magic: a Reader, London-New York, 2013.

17. Cit. en HOMANS, G.C.: "Anxiety and Ritual: the theories of Malinowsky and Raddclife-Brown", American Anthropologist, 43, 2, 1941, 164-172.

18. WITTGENSTEIN, L.: Observaciones a La rama dorada de Frazer, Madrid, 2008, 56.

19. SIGRIST, M.: "Magic and Human Reason", SHAKED, S. (ed.): Officina magica: essays on the practice of magic in Antiquity, Leiden, 2006, 293-316; GRAF, F.: "How to cope with a difficult life: a view on ancient magic", SCHAFER, P., y KIPPENBERG, H.G. (eds.): Envisioning magic. A Princeton Seminar and Symposium, Leiden-New York, 1997, 93-114; ALFAYÉ, S.: "Fraudes sobrenaturales: crédulos, impostores y potencias sobrenaturales en la Antigua Roma”, MARCO, F., PINA, F., y REMESAL, J. (eds.): Fraude, mentira y engaño en el mundo antiguo, Barcelona, 2014, 65-96. 
expresa C. Mongardini, "ante la complejidad y la presión del mundo real, se activa el pensamiento mágico para que el individuo recupere con su imaginación el control de la realidad que ha perdido" ${ }^{20}$. De acuerdo con este planteamiento, tanto la religión como la magia serían estrategias efectivas de gestión del temor capaces de generar emociones y experiencias positivas en los actores rituales mediante la combinación de la acción performativa y simbólica del ritual, la expectativa en su eficacia, el poder de la autosugestión humana, y la confianza en la intervención (beneficiosa) de las potencias sobrenaturales invocadas, y en que sus efectos pueden alcanzar una escala individual, comunitaria, e incluso cósmica. Que la creencia religiosa y la práctica ritual son mecanismos ansiolíticos eficaces contra el miedo a la muerte, la angustia existencial, y las situaciones de crisis personal y social, parece quedar demostrado por los recientes estudios de Neurociencia: el ritual disminuye, aunque sea temporalmente, la ansiedad y la indefensión existencial de los participantes, difiere la amenaza, y genera seguridad y bienestar ${ }^{21}$. Como sintetizara D.S. Whitley, "religion 'works' because of the way rituals make people feel" ${ }^{\prime 2}$.

Sin embargo, la magia y la religión también poseen su reverso tenebroso, tanto en el mundo antiguo como en el presente. De hecho, la puesta en práctica de esas estrategias contra el temor puede provocar, paradójicamente, la aparición de otras ansiedades que condicionan igualmente, y para mal, la existencia de individuos y comunidades.

Si inicialmente el rito resulta un mecanismo funcional frente al temor, su propia praxis también puede conllevar el surgimiento de nuevos miedos. Así, por ejemplo, en tanto que la confianza en su eficacia se sustenta sobre la base de un conocimiento sagrado específico y una ejecución escrupulosa de los procedimientos rituales -saber cómo y en qué condiciones- que permitan al actor concitar la atención divina y establecer un fructífero canal de comunicación, toda acción mágico-religiosa lleva en sí misma aparejada el temor a su realización inadecuada, y a que eso suponga no solo su fracaso -entendido como ineficacia ${ }^{23}$-, sino también el enfado de las potencias

20. MONGARDINI: Miedo y sociedad..., 38-40.

21. IRWIN, H.J.: "Belief in Paranormal and a Sense of Control over Life", European Journal of Parapsychology, 15, 2000, 68-78; MONGARDINI: Miedo..., 48; ESLER: “La muerte de Jesús...", 178-183; JONG, J., BLUEMKE, M, y HABLBERSTADT, J.: "Fear of Death and Supernatural Beliefs: Developing a New Supernatural Belief Scale to Test the Relationship", European Journal of Personality, 27, 5, 2013, 495-506; ALFAYÉ: “Fraudes sobrenaturales..., 65-96.

22. WHITLEY, D.S.: "Cognition, Emotion and Belief: First Steps in an Archaeology of Religion", WHITLEY, D.S., y HAYS-GILPIN, K. (EDS.): Belief in the Past. Theoretical Approaches to the Archaeology of Religion, Walnut Creek, 2008, 85-104 (98).

23. Cf. HÜSKEN, V. (ED.): When rituals go wrong: mistakes, failures and the dynamics of rituals, Leiden-Boston, 2007; SAX, W.S., QUACK, J., y WEINHOLD, J. (EDS.): The problem of ritual efficacy, 
sobrenaturales, con consecuencias catastróficas en el microcosmos personal y/o en el macrocosmos social. Pero, además, dado que el bienestar y la seguridad son estados emocionales precarios, su mantenimiento pudo hacerse depender de su continua re-actualización a través de la práctica ritual, que además debía ser intachable desde una perspectiva operacional. Ello pudo devenir en una estrategia de supervivencia hipertrofiada y por tanto patológica, creando un círculo vicioso difícil de romper y que provocaba más tensión y angustia psíquica en los participantes, que a su vez debían ser gestionadas y aliviadas a través de la complejización y/o la intensificación de rituales ya existentes, o de la creación de otros nuevos. A esos temores se une el peligro que representa la impureza y la contaminación ritual, que pueden ser neutralizadas mediante la ejecución de hábitos rituales de purificación, los cuales podían, igualmente, acabar convirtiéndose en prácticas obsesivas, disfuncionales y tremendamente ansiógenas ${ }^{24}$.

Además, aunque liberaban de algunos temores, los sistemas religiosos antiguos también educaban en el miedo y enseñaban a temer. Para empezar, a los propios dioses: la deisidaimonia o el timor deorum ${ }^{25}$ generaban un angustioso y permanente miedo a desatar su ira con nefastas consecuencias, ya fuera por desatender las obligaciones religiosas y/o no cumplir el acuerdo establecido con la divinidad; por no respetar los códigos de comportamiento social y ético impuestos por los dioses; o por cometer actos de impiedad, entre otros. Se producía, entonces, la ruptura del orden cósmico - de la pax deorum en el horizonte cultural romano-, cuyo restablecimiento iba ligado a la realización de prácticas rituales específicas de las que dependía la propia supervivencia de la comunidad implicada. De hecho, como expone J.A. Delgado en su contribución a este número, sabemos que en momentos extraordinariamente críticos de la Roma republicana (IV-I a.C.), los dioses exigieron el sacrificio de los principales magistrados del estado a cambio de la preservación de la comunidad cívica. En su trabajo "Caput in iecore non fuit. La 'cabeza' de los cónsules por la salvación de la República”, Delgado analiza minuciosamente los once casos atestiguados

Oxford, 2010; KOUTRAFOURI, V.G., y SANDERS, J. (EDS.): Ritual Failure. Archaeological Perspectives, Leiden, 2013; ALFAYÉ: “Fraudes sobrenaturales...", 70, 71, 85, 89-91; EAD., en este mismo volumen.

24. Vid., por ejemplo, DOUGLAS, M.: Purity and Danger. An analysis of the concepts of pollution and taboo, London-New York, 1989; PARKER, R.: Miasma: Pollution and Purification in Early Greek Religion, Oxford, 1991; FREUD, S.: Tótem y tabú, Madrid, 2011; LENNON, J.L.: Pollution and Religion in Ancient Rome, Cambridge, 2013; ALFAYÉ: Homo Timens..., e.p.

25. Cf., entre otros, CHANIOTIS, A.: "Constructing the fear of the gods: epigraphic evidence from sanctuaries of Greece and Asia Minor", CHANIOTIS, A. (ed.): Unveiling Emotions. Sources and Methods for the Study of Emotions in the Greek World, Stuttgart, 2012, 205-234; MATRICON-THOMAS, E.: "Le peur des dieux à Athènes pendant la Guerre du Péloponnèse: superstition et scrupules religieux", LONGERAY, S., y VALLERAT, D. (EDS.): Peurs Antiques, Saint-Étienne, 2015, 189-202. 
en las fuentes literarias antiguas en los que el animal sacrificado durante la consulta adivinatoria de los cónsules presenta una fisura en la cabeza del hígado (caput iecoris), o directamente carece de ella. Todas estas consultas se produjeron en situaciones críticas de la historia de Roma (y de los propios magistrados), en contextos de guerra y/o crisis interna en los que la angustiada y aterrorizada población solicitaba ayuda a los dioses (Polibio III, 12, 6-9) para asegurar su supervivencia. La respuesta de estos se expresaba mediante ese funesto signum, que materializaba la ruptura de la pax deorum e indicaba cuál era el procedimiento ritual que permitía su restablecimiento y la salvación de la Res Publica: el sacrificio de los cónsules, que expiaban en su persona la cólera de los dioses y cuya muerte, que debía producirse dentro del limitado tiempo de ejercicio de su magistratura, evitaría las calamidades que se cernían sobre el pueblo de Roma, librándolo del pánico y de la peligrosa amenaza que representaban sus enemigos.

Por otro lado, si bien es cierto que las narrativas escatológicas de algunas culturas del mundo antiguo ofrecían la posibilidad de la supervivencia tras la muerte, liberando al individuo de su terror mortis, también llevaban aparejadas el temor a un allende plagado de monstruos, y/o al castigo en el Más Allá por los comportamientos en vida o los modos de morir que, desde esa imaginación socio-religiosa, resultaban inapropiados $^{26}$. Asimismo, los sistemas religiosos de la Antigüedad crearon poderosas y aterradoras entidades divinas, seres mitológicos y demones que encarnaban el mal sin paliativos, figuras amenazantes que provocaban espantos sobrenaturales y frente a los cuales, nuevamente, se recurría a la práctica ritual como un mecanismo de gestión del miedo y obtención de seguridad ${ }^{27}$. Parece, por tanto, que si bien el miedo convirtió a los humanos en seres religiosos, las estrategias desarrolladas para

26. Cf., por ejemplo, BERNABÉ, A.: "Los terrores del Más Allá en el mundo griego. La respuesta órfica”, DÍEZ DE VELASCO (ED.): Miedo y religión, Madrid, 2002, 321-329; MOLINERO, M.A.: "La Dat egipcia: de preámbulo de la resurrección en el cielo a sede de suplicios eternos", DÍEZ DE VELASCO (ED.): Miedo y religión, Madrid, 2002, 303-320; ALFAYÉ, S.: "Sit tibi terra gravis: magical-ritual measures against restless dead in the Ancient World", MARCO, F., PINA, F., y REMESAL, J. (eds.): Formae mortis: el tránsito de la vida a la muerte en las sociedades antiguas, Barcelona, 2009, 181-216.

27. Vid., entre otros, MCDONOUGH, C.M.: "Carna, Proca and the Strix on the Kalends of June", Transactions of the American Philological Association, 127, 1997, 315-344; STRAMAGLIA, A.: Res inauditae, incredulae. Storie di fantasma nel mondo greco-latino, Bari, 1999; FRANKFURTER, D.: Evil Incarnate. Rumors of Demonic Conspiracy and Satanic abuse in History, Princeton-Oxford, 2006; LUGLI, U.: Umbrae. La rappresentazione dei fantasma nella Roma antica, Genova, 2008; OGDEN, D.: Night's Black Agents. Witches, Wizards and the Dead in the Ancient World, London, 2008; VERNANT, J.P.: La muerte en los ojos. Figuras del otro en la Antigua Grecia, Barcelona, 2009; SPAETH, B.S.: "The Terror that Comes in the Night: the Night Hag and Supernatural Assault in Latin Literature", SCIOLI, E., y WALDE, C. (eds.), Night-time Phenomena in Graeco-Roman Culture, Pisa, 2010, 231-258; SCHNEIDER, C.: Paranormale Antiquité. La mort et ses démons en Grèce et à Rome, Paris, 2011; PATERA: Figures grecques..., 2015. 
controlarlo -i.e., la religión y la magia-, no evitaron (sino que en ocasiones incluso propiciaron) que estos siguieran siendo temerosos.

$\mathrm{Y}$ es que los casos mencionados en absoluto agotan las dimensiones menos positivas de las interrelaciones entre temor, experiencia religiosa y práctica ritual en el mundo antiguo. Así, por ejemplo, sabemos que en la Antigüedad los operantes mágicos querían provocar pánico en sus víctimas mediante la publicidad de sus actividades, para que, por el hecho de saberse objeto de encantamiento, se generaran en ellas emociones y reacciones psicosomáticas incapacitantes que contribuyeran al éxito de la eficacia dañina de sus prácticas ${ }^{28}$. Sin embargo, su capacidad para provocar terror, sufrimiento y desesperanza entre sus víctimas, no evitó que los actores mágicos sufrieran también otros miedos y ansiedades derivados de sus propias operaciones, como estudia S. Alfayé en su contribución sobre "Los temores del mago: miedos en torno a la acción mágica en la antigua Roma”. En su trabajo se analiza cómo los practicantes de magia del Imperio Romano no estuvieron libres de sentir inquietud ante las consecuencias y las figuras amenazantes surgidas en el marco de su propia praxis mágica. Entre los variados e incluso contradictorios temores e incertidumbres que padecieron los magos de la Roma imperial, se incluyen la preocupación por el fracaso de la performance ritual y por la desactivación del artefacto mágico; la angustia ante la irreversibilidad de los efectos de su acción mágica y el recelo ante sus posibles secuelas; el miedo al ataque de las potencias sobrenaturales invocadas; la inquietud por ser descubiertos y castigados; y el temor a los rumores populares que los señalaran como hacedores de magia, entre otros.

Asimismo, sabemos que algunos "ritos de terror" - utilizando la expresión de Whitehouse ${ }^{29}$ - también fueron diseñados y ejecutados para provocar emociones aterradoras y perdurables entre los participantes, constituyendo el miedo un elemento fundamental de esas experiencias religiosas ${ }^{30}$. Es decir, que la vivencia traumática y perdurable del pánico experimentado en esas ceremonias, de naturaleza variada, sería el producto calculado y buscado de la acción ritual. En este sentido, L.H. Martin ha identificado como "ritos de terror" con iniciaciones traumáticas los rituales mis-

28. ALFAYÉ, S.: "Unexpected Contexts: revisiting the "other places" of deposition of defixiones in the Roman West", GORDON, R., MARCO, F., y PIRANOMONTE, M. (eds.), Choosing Magic, Rome, e.p.; ALFAYÉ, S.: Homo Timens..., e.p.

29. WHITEHOUSE, H.: Arguments and Icons. Divergent modes of religiosity, Oxford, 2000, 18-33.

30. Cf., entre otros, LOUCAS, I.: "Ritual surprise and terror in Ancient Greek Possession-Driven", Kernos, 2, 1989, 97-104; WHITEHOUSE: Arguments and Icons..., 18-33; ID: “Terror", CORRIGAN, J. (ed.), The Oxford Handbook of Religion and Emotion, Oxford, 2008, 259-275; DÍEZ DE VELASCO: Miedo y religión...; PETSALIS-DIOMIDIS, A.: Truly Beyond Wonders: Aelius Aristides and the cult of Asklepios, Oxford, 2010; MARTIN, L.H: Deep History, Secular Theory. Historial and Scientific Studies of Religion, Boston-Berlin, 2014, 210-212, 277-278, 327-333. 
téricos greco-romanos, y también las estancias de monjes cristianos en el desierto ${ }^{31}$, de cuyo estudio se ocupa C. Martínez Maza en esta monografía. También pudieron celebrarse ceremonias destinadas a crear temor en sus participantes en algunas de las cuevas-santuario ibéricas y mayas estudiadas en este volumen, respectivamente, por C. Rueda y J.P. Bellón, y por H. Moyes, unos espacios de culto subterráneos que constituían per se una escenografía ritual atemorizante.

De hecho, estas tres contribuciones están dedicadas al estudio de las prácticas religiosas celebradas en tres tipos de topografías del temor (fearscapes) del mundo antiguo: las cuevas, las ruinas, y el desierto. Este concepto procede de la obra de Y.F. Tuan, quien en 1979 desarrollaba en Landscapes offear ${ }^{32}$ un estudio pionero sobre los "paisajes del miedo", espacios psico-materiales de temor que son resultado de la combinación de la percepción cultural y de la experiencia psico-somática de entornos físicos tangibles. Cada cultura, cada época histórica, posee sus propias "topografías del temor", que están asociadas a la sensación de amenaza pero también a la búsqueda de protección, y que responden a su imaginación sociológica y a su cosmovisión religiosa en tanto que, como señalara D.W. Meinig, "any landscape is composed not only of what lies before our eyes but of what lies within our heads"33. Así, por ejemplo, en la antigua Roma los espacios funerarios eran considerados loca religiosa en los que era posible la interacción entre los vivos y los muertos, pero también lugares habitados por siniestros fantasmas y muertos inquietos, y por ello esas topografías liminales suscitaban ambivalentes emociones y prácticas rituales ${ }^{34}$. Y lo mismo sucedía en otros espacios transicionales de la Antigüedad en los que también podía producirse el contacto, casual o intencionado, con lo sobrenatural, como eran las encrucijadas, las puertas, y otros espacios del "cosmos de lo entreabierto" ${ }^{35}$.

En la Antigüedad, también las ruinas fueron percibidas y experimentadas como fearscapes, paisajes de la desolación cuya dinámica materialidad en descomposición reflejaba y simbolizaba la propia decadencia humana y la muerte (Artemidoro, Onei-

31. MARTIN: Deep History..., 333.

32. TUAN, Y.-T: Landscapes of fear, New York, 1979.

33. MEINIG, D.W.: The interpretation of ordinary landscapes: geographical essays, Oxford, 1979, 34.

34. ALFAYÉ: "Sit tibi...”, 181-216; EAD.: Homo timens..., e.p.

35. Vid., por ejemplo, JOHNSTON, S.I.: "Crossroads”, ZPE, 81, 1991, 217-224; ZOGRAFOU, A.: Chemins d'Hecate. Portes, routes, carrefours et autres figures de l'entre-deux. Kernos Supplément, 24, Liége, 2010; OGLE, M.B.: "The House-Door in Greek and Roman Religion and Folk-Lore", The American Journal of Philology, 32, 3, 1911, 251-271; MARCOS CASQUERO, M.A.: "Ritos y creencias de la antigua Roma relacionados con las puertas", Revisa de Estudios Latinos, 5, 2005, 147-154; FELDT, L.: "Monstrous figurines from Mesopotamia. Textuality, Spatiality and Materiality in Rituals and Incantations for the Protection of Houses in First-Millenium Assur", BOSCHUNG, D., y BREMMER, J.N. (eds.), The materiality of magic, Paderborn, 2015, 59-96; ALFAYÉ: “Unexpected contexts..., e.p. 
rocritica IV, 30) ${ }^{36}$. Además, esos espacios estaban habitados por amenazantes seres sobrenaturales, como muestran las desagradables experiencias de los encuentros con ellos recogidas en las fuentes literarias, lo que convertía a las ruinas en las topografías idóneas para la celebración de rituales religiosos y mágicos que persiguieran explotar su potencialidad transicional ${ }^{37}$. De hecho, una de las contribuciones de este volumen monográfico está dedicada al estudio de dos poderosas topografías del miedo en la imaginación y la praxis religiosa del cristianismo egipcio de época tardía: las ruinas de los templos paganos y el paisaje desolado del desierto. Como analiza C. Martínez Maza en su artículo "Fearscapes cristianos en el Egipto tardoantiguo", el desierto fue concebido como un peligroso espacio liminal que servía de refugio a los dioses paganos, transformados ya en demonios que poblaban los antiguos santuarios y que atacaban y aterrorizaban a los monjes que se adentraban en ellos con ruidos, gritos, y violencia física. Los templos en ruinas y el paisaje desértico egipcio constituían la ominosa escenografía en la que se desarrollaba el encuentro del cristiano con los demonios, una terrorífica pero crucial experiencia religiosa para el monje, que debía confrontar y derrotar al diablo en ese fearscape como un "rito de paso", como una prueba iniciática que le permitía completar su progreso espiritual en el recorrido monástico. Además, estos relatos ofrecían a su audiencia información valiosa sobre las temidas artimañas del Maligno, y sobre cómo era posible protegerse de él y vencerlo siguiendo el ejemplo virtuoso de los monjes en sus encuentros en el desierto.

Los trabajos de C. Rueda y J.P. Bellón, y el escrito por H. Moyes abordan el análisis de las cuevas como otro fearscape religioso del mundo antiguo, y el estudio de las verenda numina y de las prácticas rituales documentadas en ellas. Esos fearscapes subterráneos resultaban somáticamente hostiles y perturbadores para los actores rituales que los frecuentaban en tanto que adentrarse por ellos implicaba transitar por un paisaje completamente diferente al ordinario, lo que provocaba intensas respuestas emocionales de miedo y angustia, pero también de reverencia antes las potencias sobrenaturales que moraban en el inframundo, con las que se buscaba establecer un contacto tan deseado como temido a través de estrategias rituales diversas ${ }^{38}$.

36. GINSBERG, R.: The Aesthetics of Ruins, Amsterdam-New York, 2004; EDENSOR, T.: Industrial ruins: space, aesthetics \& materiality, Oxford-New York, 2005.

37. Cf., por ejemplo, NARDI, E.: Case "infestati da spiriti" e diritto romano e moderno, Milán, 1960; PERCIVAL, J.: "Saints, ghosts and the Afterlife of the Roman Villa", L'Antiquité Classique, 65, 1996, 161173; FELTON, D.: Haunted Greece and Rome. Ghost Stories from Classical Antiquity, Austin, 1999; ALFAYÉ: “Unexpected contexts..., e.p.

38. Sobre la frecuentación religiosa de cuevas en el mundo antiguo, vid. LAVAGNE, H.: Operosa Antra. Recherches sur la grotte à Rome de Sylla à Hadrien, Roma, 1988; USTINOVA, Y.: Caves and the Ancient Greek Mind. Descending Underground in the Search for Ultimate Truth, Oxford, 2009; ALFAYÉ, S.: "Hacia el lugar de los dioses: aproximación a la peregrinación religiosa en la Hispania indoeuropea", 
En su artículo "Culto y rito en cuevas: modelos territoriales de vivencia y experimentación de lo sagrado, más allá de la materialidad (ss. V-II a.n.e.)", Rueda y Belón exploran las variadas dinámicas rituales celebradas en cuevas de la zona del Alto Guadalquivir por las poblaciones ibéricas, relacionándolas con los procesos históricos locales y los modelos de organización territorial en los que se integran, así como con diferentes narrativas míticas. Además, analizan cómo la escenografía ritual y la intencionada modificación antrópica de algunas de estas cavidades estuvieron destinadas a provocar emociones y respuestas religiosas específicas en los participantes, entre las que se incluye el temor reverencial ante la hierofanía, y a controlar el grado de accesibilidad y comunicación con lo divino. Por otra parte, Rueda y Bellón interpretan las heterogéneas prácticas cultuales celebradas en esas cuevas como estrategias ritualizadas de gestión del miedo por parte de las comunidades ibéricas. Desde esta perspectiva, proponen identificar algunas acciones cultuales colectivas como prácticas de agregación que restablecían los vínculos sociales e identitarios, mientras que otros rituales estarían destinados a propiciar la fecundidad de la naturaleza, asegurando así la supervivencia del grupo y disminuyendo su ansiedad. También los individuos que debían afrontar peligrosos procesos transformativos asociados a ritos de paso habrían encontrado protección y seguridad por medio de las performances realizadas en esas cuevas-santuario, y otros pudieron canalizar su miedo a la enfermedad, el dolor y/o la muerte mediante diversos rituales curativos.

$\mathrm{Al}$ igual que sucede en el mundo ibérico, en la cultura maya del período clásico también se documentan diversas dinámicas de culto celebradas en las entradas y el interior de las cuevas, como analiza H. Moyes en su contribución sobre "Xibalba, the Place of Fear: Caves and the Ancient Maya Underworld". Según la cosmología maya, las cuevas eran "el lugar del miedo" o Xibalba, el espacio subterráneo habitado por los muertos y los Señores del Inframundo, entre los que se incluyen dioses de la fertilidad y la lluvia. Ello las convertía en topografías religiosas de la ansiedad, pero también en espacios transicionales que permitían acceder al Más Allá y entrar en contacto con divinidades temidas pero de las que dependía la supervivencia de la comunidad, y que podían ser aplacadas mediante ceremonias y prácticas rituales, tanto individuales como colectivas, entre las que se incluyen la ofrenda de cerámicas, objetos domésticos, joyas e incienso, y el sacrificio de víctimas animales y humanas.

MARCO, F., PINA, F., y REMESAL, J. (eds.), Viajeros, peregrinos y aventureros en el mundo antiguo, Barcelona, 2010, 177-218; BERGSVIK, K.A., y SKEATES, R. (EDS.): Caves in context. The cultural significance of caves and rockshelters in Europe, Oxford, 2012; MOYES, H. (ED.): Sacred Darkness. A global perspective on the ritual use of caves, Colorado, 2012; RÍSQUEZ, C., y RUEDA, C. (EDS.): Santuarios íberos: territorio, ritualidad y memoria, Jaén, 2013; DOWD, M.: The Archaeology of Caves in Ireland, Oxford, 2015; DOWD, M., y HENSEY, R. (EDS.): The Archaeology of Darkness, Oxford, 2016. 
Significativamente, se documenta un incremento de la actividad religiosa maya en las cuevas estudiadas por Moyes durante épocas de crisis ecológica y colapso social, lo que revela la importancia social de esos rituales subterráneos como mecanismos de gestión de la ansiedad individual y comunitaria, con los que se pretendía principalmente renovar y propiciar la lluvia y la fertilidad y, en último extremo, garantizar la supervivencia frente a la amenaza de la muerte y el caos.

Como se infiere de la lectura de este prólogo, este número monográfico de Arys dedicado a las "verenda numina" no constituye una síntesis de los miedos y las experiencias religiosas de la Antigüedad, sino una aproximación parcial a un tema complejo y apenas explorado historiográficamente a través de diversos estudios de caso sobre temores, emociones, fearscapes, y rituales mágicos y/o religiosos documentados en ámbitos culturales, geográficos y cronológicos muy dispares del mundo antiguo. Indudablemente, muchos temas no han sido abordados aquí. Por ejemplo, el de los dioses que encarnaban aspectos diversos del temor, como el griego Phóbos ${ }^{39}$, o los romanos Pallor y Pavor (Livio I, 27; Serv., Aen. 8, 285). O el de la variada cultura material del miedo y la protección mágico-religiosa, cuyo estudio debería incluir tanto la tipología de imágenes y artefactos utilizados para generar seguridad o provocar el pánico, como un análisis de su selectiva distribución topográfica. Tampoco se ha abordado aquí la relación entre política, religión y miedo ${ }^{40}$. Ni, entre otras cuestiones, la explotación fraudulenta y comercial de los temores, ansiedades y esperanzas de individuos y comunidades por parte de falsarios religiosos y mágicos, que lograban embaucar a su audiencia mediante una "trampa sagrada" que los cautivaba sensorial y emocionalmente ${ }^{41}$.

Hay, sin duda, muchos más aspectos de las complejas conexiones entre el miedo, la experiencia religiosa y la praxis ritual en las sociedades del pasado que merecen ser estudiados. Como editora de este volumen, espero que al menos los estudios recogidos aquí sirvan para mostrar la potencialidad de esta línea de investigación, infravalorada por la historiografía hasta fechas muy recientes, y permitan avanzar en el conocimiento de las poliédricas relaciones entre miedo y religión, tanto en el

39. KANOVOU, N.: "Negative emotions and Greek Names", CHANIOTIS, A., y DUCREY, P. (eds.): Unveiling Emotions II. Emotions in Greece and Rome: texts, images, material culture, Stuttgart, 2013, 167-190 (178); PATERA, M.: "Reflections on the discourse of fear in Greek sources", CHANIOTIS, A., y DUCREY, P. (eds.): Unveiling Emotions II. Emotions in Greece and Rome: texts, images, material culture, Stuttgart, 2013, 109-134; HÂNCU, M.-C.: "Phobos and its Place in Hesiod's Theogony", DUMITRU, M.L., HALICHIAS, A.C., y POPA, N.A. (EDS.): Expressions of Fear from Antiquity to the Contemporary World, Newcastle-upon-Tyne, 2016, 11-20.

40. Vid. ALFAYÉ: Homo Timens..., e.p.

41. ALFAYÉ, S.: "Fraudes sobrenaturales...", 65-96. 
pasado como en nuestro propio e incierto presente. Solo quisiera, por último, agradecer a J.R. Carbó, secretario de la revista $A R Y S$, su esfuerzo para llevar a buen puerto este número, y a los autores por sus interesantes contribuciones; sin todos ellos, esta monografía no habría sido posible. 